\title{
Exercise-Induced Extracellular Vesicles Delay the Progression of Prostate Cancer
}

\author{
Lilite Sadovska $^{1}$, Jānis Auders ${ }^{1,2}$, Laura Keiša ${ }^{1,2}$, Nadezhda Romanchikova $^{1}$, \\ Laila Silamikele $^{3}$, Madara Kreišmane ${ }^{3}$, Pawel Zayakin ${ }^{1}$, Satoru Takahashi ${ }^{4}$, Zane Kalniṇa ${ }^{3+}$ \\ and Aija Liné ${ }^{1,5 * t}$
}

${ }^{1}$ Cancer Biomarker Group, Latvian Biomedical Research and Study Centre, Riga, Latvia, ${ }^{2}$ Faculty of Medicine, University of Latvia, Riga, Latvia, ${ }^{3}$ Laboratory Animal Core Facility, Latvian Biomedical Research and Study Centre, Riga, Latvia, ${ }^{4}$ Department of Experimental Pathology and Tumor Biology, Nagoya City University Graduate School of Medical Sciences, Nagoya, Japan,

${ }^{5}$ Faculty of Biology, University of Latvia, Riga, Latvia

Increasing evidence suggests that regular physical exercise not only reduces the risk of cancer but also improves functional capacity, treatment efficacy and disease outcome in cancer patients. At least partially, these effects are mediated by the secretome of the tissues responding to exercise. The secreted molecules can be released in a carrier-free form or enclosed into extracellular vesicles (EVs). Several recent studies have shown that EVs are actively released into circulation during physical exercise. Here, we for the first time investigated the effects of exercise-induced EVs on the progression of cancer in an F344 rat model of metastatic prostate cancer. Although we did not observe a consistent increase in the circulating EV levels, RNA sequencing analysis demonstrated substantial changes in the RNA content of EVs collected before and immediately after forced wheel running exercise as well as differences between EVs from runners at resting state and sedentary rats. The major RNA biotype in EVs was mRNA, followed by miRNA and rRNA. Molecular functions of differentially expressed RNAs reflected various physiological processes including protein folding, metabolism and regulation of immune responses triggered by the exercise in the parental cells. Intravenous administration of exercise-induced EVs into F344 rats with orthotopically injected syngeneic prostate cancer cells PLS10, demonstrated reduction of the primary tumor volume by $35 \%$ and possibly - attenuation of lung metastases. Hence, our data provide the first evidence that exercise-induced EVs may modulate tumor physiology and delay the progression of cancer.

Keywords: extracellular vesicles, exercise, prostate cancer, RNA cargo, RNA sequencing

\section{INTRODUCTION}

Prostate cancer (PC) is the second most frequently diagnosed cancer in males worldwide affecting more than 1.4 million men per year. In terms of mortality, PC is the fifth leading cause of death from cancer in men (Ferlay et al., 2015). Hence, PC is a global health problem requiring effective primary, secondary, and tertiary prevention measures. Regular physical activity is associated with a lower incidence of many common types of cancer (Moore et al., 2016), whereas the association between physical activity and PC risk remains controversial. Some studies have reported that leisure-time 
physical activity is associated with a higher risk of PC, some studies showed no clear relationship, however larger number of studies have found a decrease of PC incidence in physically active men and the effect showed a dose-response relationship with vigorous activity (Shephard, 2017). Furthermore, several studies have shown that exercise reduced fatigue and treatment side effects, improved quality of life, prevented disease recurrence, and improved survival of PC patients (Kenfield et al., 2011; Bourke et al., 2016; Shephard, 2017; Belloni et al., 2021). Moreover, experiments in murine tumor models have demonstrated that exercise leads to a significant reduction in tumor size and incidence, and these effects are associated with remodeling of the immune tumor microenvironment (TME) (Pedersen et al., 2016; Idorn and Thor Straten, 2017). This suggests that apart from the well-documented beneficial effects of exercise on cardiovascular fitness, energy balance and body weight, it may have a direct effect on cancer. Therefore, exercise may not just be preventive but also therapeutic and hence serve as an important tool for tertiary prevention of cancer.

At least partially, the effects of exercise are mediated by various molecules (proteins, lipids, RNAs, metabolites etc.) secreted into the circulation by muscle, bone, brain, liver and other tissues. These molecules can be secreted in a soluble form or packaged into carriers such as extracellular vesicles (EVs). The term "EVs" refers to all kinds of vesicles naturally released from cells that are delimited by a lipid bilayer and cannot replicate (Thery et al., 2018). According to the mode of biogenesis, three main types of EVs have been defined: exosomes, microvesicles and apoptotic bodies (Yanez-Mo et al., 2015). They differ in their molecular content, size, membrane composition, cellular source and specific functions. Although initially considered to be a waste disposal mechanism (Johnstone et al., 1987), it is now clear that EVs generated by both live and apoptotic cells interact with the recipient cells and are important mediators of intercellular communication (Tkach and Thery, 2016; Caruso and Poon, 2018). EVs can be internalized by the recipient cells and trigger various intracellular signal transduction pathways (Yanez-Mo et al., 2015; Zhan et al., 2021) or bind to the cell surface receptors and trigger the respective downstream signaling pathway (Muller et al., 2016; Muller et al., 2017).

Exercise has been shown to induce a rapid release of EVs into circulation (Fruhbeis et al., 2015; Whitham et al., 2018). A recent study demonstrated that exercise induced the secretion of over 300 proteins into EVs, including glycolytic enzymes and myokines, i.e. cytokines secreted by skeletal muscle (Whitham et al., 2018). Myokines have been shown to act as tumor suppressors and may impact several hallmark features of cancer (Hojman et al., 2011; Ruiz-Casado et al., 2017), whereas glycolytic enzymes might induce changes in the metabolic activity of cancer cells (Pedersen et al., 2015). We hypothesized that exercise-induced EVs may directly interact with cancer cells and alter their behavior as well as change the functional phenotype of circulating and tumor-infiltrating immune cells thus affecting the growth rate and metastatic potential of cancer. In this pilot study, we investigated the RNA content of EVs released during forced wheel running and their effects on the progression of cancer in a rat model of metastatic prostate cancer.

\section{MATERIALS AND METHODS}

\section{Animal Care and Experimental Design}

The experimental procedures in animals were approved by the National animal welfare and ethics committee (permit no. 121/ 2021) and were performed in compliance with the Directive 2010/ $63 / \mathrm{EU}$ as adopted in the national legislation.

In total, 37 naïve SPF male Fischer 344 rats were obtained from Charles River Laboratories, Germany (immunocompetent inbred strain F344/DuCrl). During the introduction, animals were randomly allocated in cages in pairs or trios; individually ventilated cages GR900, HEPA-ventilated by SmartFlow air handling unit (Tecniplast, Italy) at 75 air changes per hour were used for animal housing. Access to autoclaved water acidified to $\mathrm{pH} 2.5-3.0$ with $\mathrm{HCl}$ and standard rodent diet (4RF21 (A), Mucedola) was provided ad libitum. Aspen wooden bedding and nesting material (Tapvei, Estonia) together with rat cardboard houses (Velaz, Czech Republic) and aspen gnawing bricks (Tapvei, Estonia) were provided in all cages, and cages were changed every 7 days. Animals were housed in SPF facility under controlled temperature $\left(24 \pm 1^{\circ} \mathrm{C}\right)$ and relative humidity of $40-60 \%$. Animal health monitoring was performed in line with FELASA recommendations (Mähler et al., 2014).

All animals were subjected to at least 2-weeks acclimatization period with the adaptation to a reverse $12 \mathrm{~h}$ light/dark cycle (dark phase set to 10:00 am-10:00 pm; visible light intensity $<25$ lux), and then used to model either regular physical exercise (i.e. forced wheel running model) or orthotopic PC development [PLS10 rat PC model (Suzuki et al., 2015)]-see Figure 1 for schematic overview. An individual animal served as an experimental unit in both models. Before starting the procedures, the animals were identified by tattooing their tails using AIMS $^{\mathrm{TM}}$ NEO-9 Neonate Tattooing System according to the manufacturer's instructions.

\section{Cell Culture}

Rat prostate cancer cell line PLS10 was developed previously using chemically-induced prostate carcinoma in F344 rats (Nakanishi et al., 1996; Suzuki et al., 2015). Cells were maintained in RPMI-1640 medium, supplemented with 10\% FBS, $2 \mathrm{mM}$ L-glutamine and $1 \mathrm{x}$ Antibiotic-Antimycotic at $+37^{\circ} \mathrm{C}$ in a humidified atmosphere containing $5 \% \mathrm{CO} 2$.

Cells in the exponential growth phase were trypsinized, counted and resuspended in PBS in aliquots of $5 \times 10^{6}$ cells per $25 \mu \mathrm{L}$ of PBS. The aliquots were immediately transferred to the animal facility and kept on ice water until injection. Just before injection into the rat prostate, the cell suspension was mixed with an equal volume of ice-cold Matrigel (Corning \#356237, USA). Fresh cell suspensions were prepared for every batch of 3-4 animals undergoing laparotomic surgery.

\section{Forced Wheel Running Exercise}

In total, 16 7-week-old male F344 rats were subjected to model regular exercise and sedentary lifestyle. The number of animals per group $(n=8)$ was chosen based on the available data on average EV plasma concentrations and calculations we made to ensure the necessary amount of EVs for the 6-weeks injection 

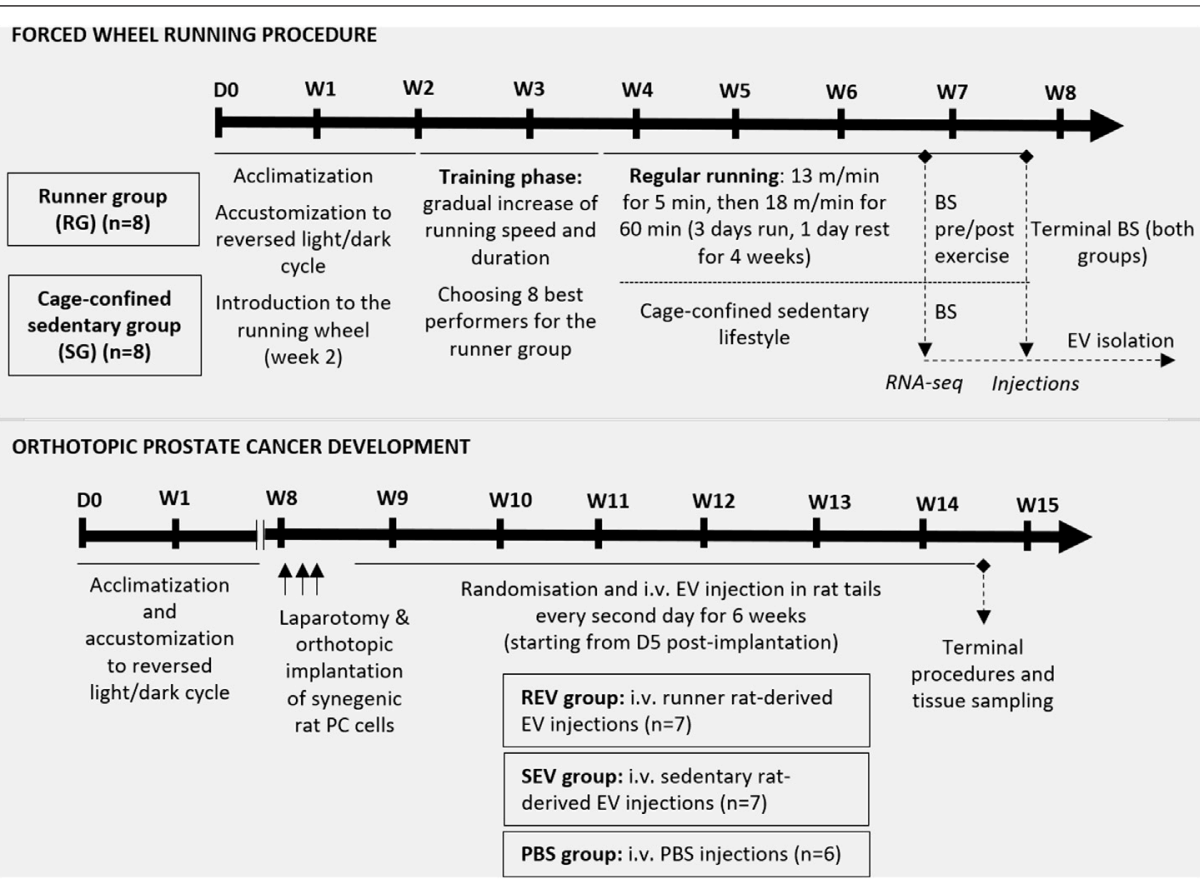

FIGURE 1 | Schematic overview of the in vivo study design. The upper panel represents the steps of the forced wheel running procedure-in total, sixteen $7 \mathrm{w} / \mathrm{O}$ male F344 rats underwent a 2-weeks acclimatization period. During the second week, rats were introduced to the running wheel, which was followed by a 12-days training phase for all experimental animals. During the training phase, 8 best performers were chosen for the runner group while the remaining 8 rats were assigned to the cage-confined sedentary group. The running exercise phase lasted for 5 weeks in total, including 4 weeks of regular running in a setting described in the figure. After 4 weeks of exercise, blood samples were taken before and shortly after $1 \mathrm{~h}$ running from the runner group and a single blood sample from the control animals for further plasma EV analyses. At the endpoint, total blood sample was taken via cardiac puncture and used for plasma EV isolation. The lower panel shows the steps of in vivo evaluation of the effect of EVs (isolated from the endpoint plasma samples) on orthotopic PC development in syngeneic male F344 rats. Briefly, anesthetized 12 w/o male F344 rats were subjected to a laparotomic incision to enable orthotopic implantation of $5 \times 10^{6} \mathrm{PLS} 10$ rat prostate cancer cells into the ventral prostate. After stratified randomization, the animals were divided into 3 groups and, starting from day 5 post-implantation, rats from all 3 groups received i.v. injection of EVs or PBS as shown. After 6 weeks of injections, animals were sacrificed, and tissues of interest were collected. BS, blood sampling; D, day; i.v., intravenous; W, week.

period in the syngeneic PC model animals (Figure 1). In the second week after arrival and acclimatization to the reversed light/dark cycle, rats were introduced to the forced running wheel (system for rats, model $80805 \mathrm{~A}$, Lafayette Instrument) without a specific running mode. It was followed by a 12-days training phase for all 16 experimental animals (started at 9 weeks of age) by gradually increasing the running speed and duration. During the training phase, 8 best performers were selected for the runner group while the remaining 8 rats were assigned to the sedentary group. After assigning to groups, the animals were kept cohoused with their initial cage mates.

The regular forced running exercise phase for the runner group lasted for 5 weeks in total, including 4 weeks of regular running in a setting described in Figure 1. After 4 weeks of regular exercise, rats underwent brief anesthesia with $1.5 \%$ isoflurane and $500 \mu \mathrm{L}$ of blood from the tail vein were taken. This step was repeated for the same animals immediately after $1 \mathrm{~h}$ of running. On the same day, a blood sample was collected from the sedentary rats following the same protocol. After 5 weeks of regular exercise, all the animals immediately after $1 \mathrm{~h}$ running were subjected to deep surgical anesthesia with $5 \%$ isoflurane and underwent terminal blood collection via cardiac puncture. Blood was immediately collected into S-Monovette Hematology EDTA
K3 tubes (Sarstedt, Germany) or BD Vacutainer ${ }^{\mathrm{TM}}$ Blood Collection Tubes with K2EDTA (Fisher Scientific, USA), depending on the volume, and centrifuged at $2000 \mathrm{x} \mathrm{g},+4^{\circ} \mathrm{C}$ for $15 \mathrm{~min}$. Plasma was collected in fresh tubes and immediately frozen for further EV isolation.

\section{Isolation of EVs}

EVs were isolated from rat plasma by size exclusion chromatography (SEC) using qEVoriginal/35 $\mathrm{nm}$ or $\mathrm{qEV} 10 /$ $35 \mathrm{~nm}$ columns (IZON, USA) depending on the plasma volume. The SEC fractions were analyzed using ZetaSizer Nano ZS (Malvern Panalytical, UK) and the fractions containing EVs were concentrated using Amicon Ultra-0.5, Ultracel-3 Membrane, $3 \mathrm{kDa}$ centrifugal filter units (Merck Millipore, Germany). EVs that were used for RNA sequencing were treated with proteinase K (Thermo Fisher Scientific, USA) and RNAse A (Thermo Fisher Scientific, USA) to remove all the free proteins and RNAs that are not enclosed in EVs. EVs were visualized by transmission electron microscopy (TEM) and the size distribution profile and concentration of EVs were determined by nanoparticle tracking analysis (NTA) using NanoSight NS500 instrument (Malvern, UK) as described before (Endzelins et al., 2017). 


\section{RNA Extraction}

RNA was extracted from plasma EVs using miRNeasy micro kit (Qiagen, USA) according to the manufacturer's protocol. The concentration and quality of the obtained RNA were analyzed on Agilent Bioanalyzer with Agilent RNA 6000 Pico chip (Agilent Technologies, Germany).

\section{RNA Sequencing and Data Analysis}

RNA libraries were constructed using CleanTag ${ }^{\circledR}$ Small RNA Library Prep Kit (Trilink Biotechnologies, USA), the quality and concentration of obtained libraries were analyzed on Agilent Bioanalyzer using Agilent High Sensitivity DNA chip (Agilent Technologies, Germany). The libraries were cleaned using Blue Pippin DNA Size Selection with 3\% gel Blue Pippin Cassette (Sage Science, USA) setting tight target length to $140 \mathrm{bp}$ thus selecting fragments with size in tight range to $140 \mathrm{bp}$ (126-154 bp). The libraries were diluted as required and sequenced on Illumina NextSeq500 instrument using NextSeq 500/550 Mid Output Kit v2.5 (150 cycles) (Illumina, USA).

The obtained raw data in fastq format were analyzed using ad-hoc $\mathrm{R}$ script pipeline, which included: adapter trimming [cutadapt (Martin, 2011)], read mapping [bowtie2 (Langmead and Salzberg, 2012)] against RGSC rat (Rattus norvegicus) genome (version Rnor_6.0), multi-aligned reads reposition [ShortStack (Axtell, 2013)], counting [Rsubread package (Liao et al., 2019)] with RGSC (version Rnor_6.0) and miRbase (Kozomara et al., 2019) annotations. For differentially expressed gene (DEG) analysis, the reads were normalized per sample, the reads mapped to features were counted and analyzed using quasi-likelihood F-tests by edgeR (McCarthy et al., 2012) package. A subset of DEGs (adj. $p<0.05$ ) was subjected to GO terms [GOstats (Falcon and Gentleman, 2007)] and enrichment analyses (rentrez (Winter 2017), GO.db (Carlson, 2019a), org.Rn.eg.db (Carlson, 2019b) packages).

\section{Syngeneic Orthotopic Prostate Cancer Model}

For modeling an orthotopic PC development, 21 F344 rats were used-the sample size of 7 animals per group (see Figure 1 for study groups) was calculated by statistical power analyses using $\mathrm{G}^{\star}$ Power software (Buchner et al., 2021) and taking into account $80 \%$ power and $\alpha=0.05$, the published tumor size variation (Suzuki et al., 2015), expected effect size and the chosen statistical test for the result analyses.

At the age of 12 weeks, each animal underwent laparotomic surgery by using an aseptic technique under $2.5 \%$ isoflurane anesthesia, and $5 \times 10^{6}$ syngeneic rat prostate cells PLS10 in $50 \mu \mathrm{L}$ total volume containing 50\% Matrigel were injected into the ventral lobe of the prostate. The incisions were closed with Novosyn 4/0 DS19 mid-term absorbable sutures (B.Braun, Germany) and secured with surgical adhesive. An ophthalmic gel was provided during surgery and $8 \mathrm{ml}$ of warm saline was administered subcutaneously immediately after surgery. Each animal received subcutaneous Meloxicam ( $1 \mathrm{mg} / \mathrm{kg})$ injection during surgery and for 3 days postsurgery. Animals were single housed in sterile cages for 3 days and then returned to their home cages. Animals were closely monitored for possible postsurgical complications; the wounds were treated with furasol solution. In total, 7 rats divided in 2 sets were implanted with PC cells per day, and animals were allocated to the study groups after stratified randomization by considering this setting (Figure 1; one animal did not survive the surgery).

Starting from day 5 post-implantation, rats from all study groups via lateral tail vein received i.v. injections of $100 \mu \mathrm{L} \mathrm{EV}$ solution containing $1.5 \times 10^{10} \mathrm{EVs}$ in PBS or PBS every second day as shown in Figure 1. During PC development, animals were carefully monitored for possible signs of suffering (following the IACUC Policy \#012), and the tumor size was estimated by palpation. After 6 weeks of EV injection, all study animals were humanely euthanized, and terminal blood samples and tissues of interest were collected and fixed in $10 \%$ buffered formalin. Primary tumors were measured, and tumor volume was calculated using the following formula: tumor volume = (width)2 $\mathrm{x}$ length/2.

\section{Statistical Analysis}

A nonparametric one-tailed Mann-Whitney test was used to compare the tumor volume and the number of metastases between groups of animals. Fisher's exact test was used to compare the number of animals with/without metastases between groups of animals. $p$-value of $\leq 0.05$ was considered to be significant. The statistical analyses were performed with GraphPadPrism 7 (GraphPad, USA).

\section{RESULTS}

\section{Effect of Forced Wheel Running Exercise on the Plasma EV Levels}

EVs were isolated from rat plasma before (Pre-RUN) and immediately after forced wheel-running exercise (Post-RUN) and the yield, size and purity of EVs were assessed by TEM and NTA. TEM images revealed that the majority of particles were ranging from 30 to $160 \mathrm{~nm}$ in diameter and had a cup-shaped morphology that is typically observed for exosomes in TEM. However, smaller particles that possibly represent lipoprotein particles and a small number of large particles of 200-250 nm in diameter were also present in the majority of the samples analyzed and we did not observe any significant differences between Pre-RUN and Post-RUN samples (Figure 2A,B). NTA showed that the major fraction of particles was in the size range from 36 to $200 \mathrm{~nm}$ and the concentrations of EVs ranged from 6.5 $\times 10^{9}$ to $1.9 \times 10^{11}$ particles per $\mathrm{ml}$ of plasma (Figure $2 \mathrm{C}$ ). However, no significant differences neither in size or concentration of EVs between Pre-RUN and Post-RUN samples were observed (Figure 2D).

\section{Changes in the EV RNA Content During Exercise}

To determine whether exercise affects the EV-enclosed RNA cargo, we performed RNA sequencing analysis of Pre-RUN $(n=8)$ and Post-RUN $(n=8)$ plasma EVs from the runner group and plasma EVs from the sedentary group $(n=8)$. Analysis of the total EV RNA by Bioanalyzer showed the dominant peaks 

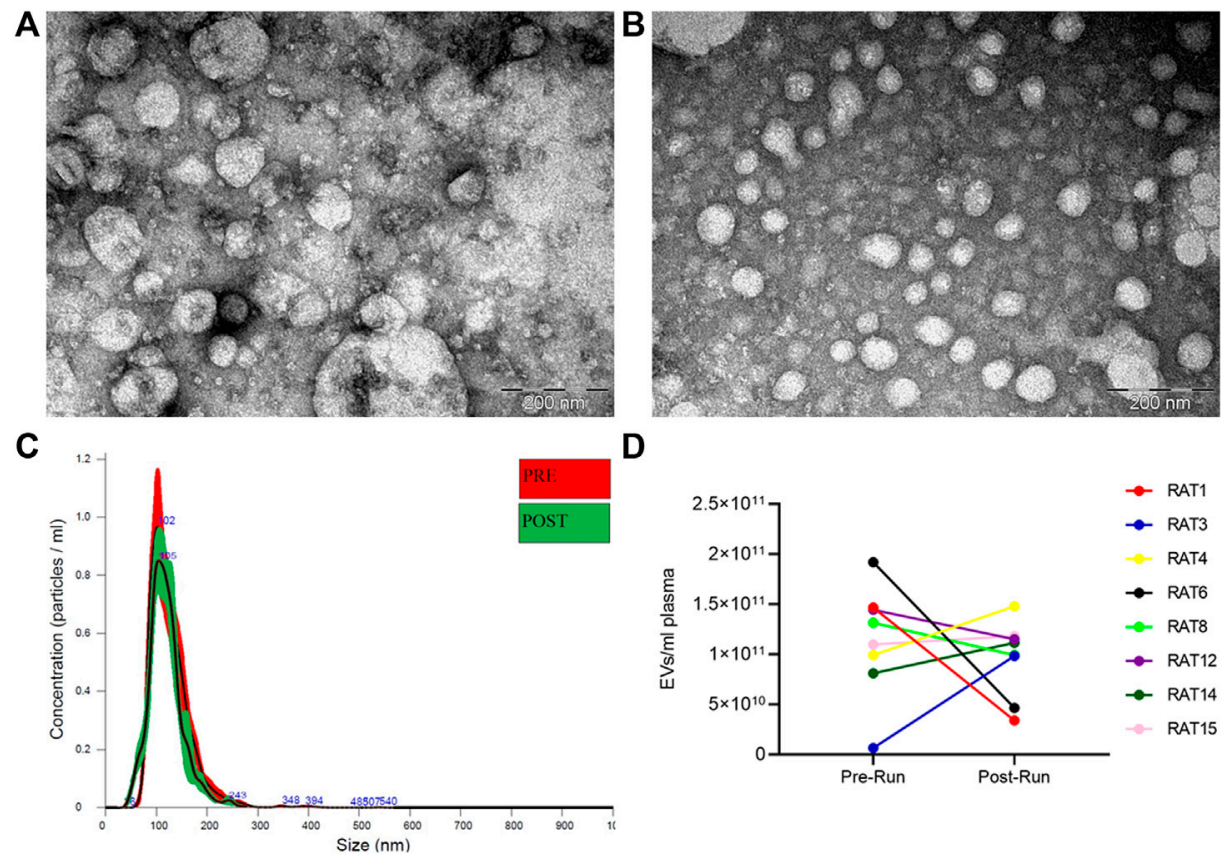

D

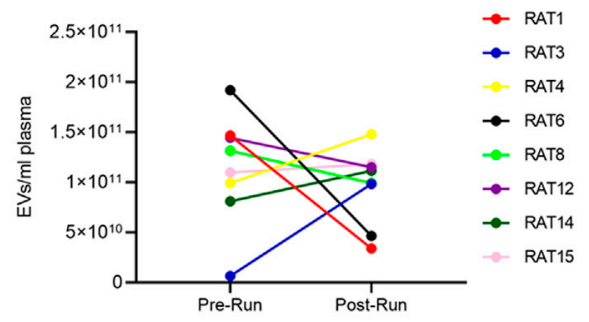

FIGURE 2 | Characteristics of EVs released in plasma during forced wheel running exercise. (A) Representative TEM image of Pre-RUN plasma EVs. (B) Representative TEM image of Post-RUN plasma EVs. (C) Quantity and size distribution of Pre-RUN and Post-RUN plasma EVs assessed by NTA (a representative case). (D) Paired dot plots show the number of particles in Pre-RUN and corresponding Post-RUN plasma samples determined by NTA.

in the range from 20 to $150 \mathrm{nt}$ (data not shown). The total EV RNA was used for the RNA-seq library construction without a prior size selection. Importantly, prior to the RNA extraction, EVs were treated with Proteinase $\mathrm{K}$ and RNase A to remove RNAs that are attached to the surface of EVs. On average, 5.9 million raw reads were obtained per library, however, on average 2.85 million reads per library remained after the quality control, adapter trimming, and filtering out the reads that were shorter than $16 \mathrm{nt}$. The average overall alignment rate to the Rattus norvegicus genome was $51.5 \%$. The aligned reads were counted using Rsubread package (Liao et al., 2019) and RGSC rat genome and genome annotation (version Rnor_6.0). Results showed that the majority of the reads were mapped to mRNAs $(73.8 \%)$, followed by miRNAs (13.6\%) and rRNAs (6.7\%), while the other biotypes-lincRNAs, mitochondrial rRNAs, processed pseudogenes etc. constituted less than $2 \%$ each. However, when the reads were counted using miRBase annotation, $0.6 \%$ of the mapped reads were counted as mature miRNAs and a total of 194 different miRNAs were identified.

Next, we performed differential expression analysis using edgeR package. RNAs which expression was detected in less than 4 samples were excluded from the analysis. To assess whether the RNA cargo of EVs that are released into the circulation during the forced wheel running exercise is distinct from that in the resting state, Post-RUN EVs were compared against the Pre-RUN EVs. A total of 20 differentially expressed genes (DEGs) (adj. $p<0.05)-10$ upregulated and 10 downregulated during the exercise were identified (Figure 3A; Supplementary Table S1). The top 10 DEGs are shown in Table 1. All DEGs are protein-coding genes and no differentially expressed RNAs were found in other RNA biotypes. GO term enrichment analysis of DEGs revealed enrichment of genes related to unfolded protein binding (adj. $p=$ 0.048).

To assess the long-term effects of exercise on the RNA cargo of circulating EVs, Pre-RUN EVs were compared to the plasma EVs from the sedentary control rats. A total of 52 genes were differentially expressed (adj. $p<0.05$ ), including 50 proteincoding genes and 2 miRNAs (Figure 3B; Supplementary Table S2). Only eleven of these RNAs had higher levels in the Pre-RUN EVs than in sedentary control EVs. The top 10 DEGs are shown in Table 2. GO term enrichment analysis showed the enrichment of genes associated with molecular functions "selenium binding" and "oxidoreductase activity, acting on peroxide as acceptor" (both adj, $p=0.025$ ).

\section{Effect of Exercise-Induced EVs on the Progression of Prostate Cancer}

Next, we investigated the effects of EVs released during the forced wheel running exercise on the progression of $p \mathrm{C}$. Briefly, total blood plasma EVs were isolated from F344 rats subjected to forced running wheel exercise or sedentary lifestyle, and the obtained EVs were subsequently administered intravenously in F344 rats with orthotopically injected syngeneic prostate cancer cells PLS10 (established from chemically induced, castrationresistant metastatic PC). The PLS10 PC rat tumor model was adopted based on the protocol originally published by the authors (Suzuki et al., 2015). PC-bearing rats received EV injections 


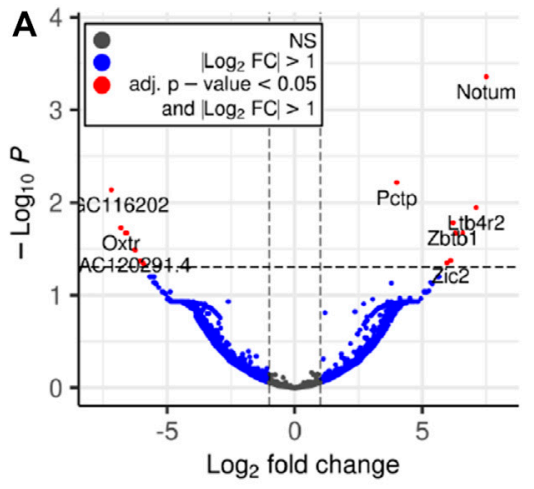

total $=13162$ variables

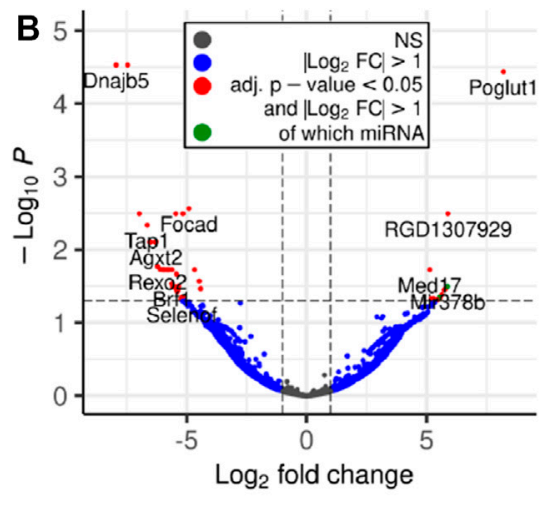

total $=12696$ variables

FIGURE 3 | Volcano plots depicting significant changes in the EV-enclosed RNA content. (A) Post-RUN vs. Pre-RUN EVs from exercised rats. (B) Pre-RUN EVs from exercised rats vs. EVs from sedentary control group rats.

TABLE 1 | Top 10 differentially expressed genes in Post-RUN vs. Pre-RUN EVs.

\begin{tabular}{|c|c|c|c|c|c|}
\hline Gene name & Description & Function Smith et al. (2020) & Expression Yu et al. (2014) & LogFC & $\begin{array}{l}\text { Adjusted } \\
p \text {-value }\end{array}$ \\
\hline Notum & $\begin{array}{l}\text { NOTUM, palmitoleoyl-protein } \\
\text { carboxylesterase }\end{array}$ & $\begin{array}{l}\text { Regulation of Insulin-like Growth Factor (IGF) transport } \\
\text { and uptake by Insulin-like Growth Factor Binding } \\
\text { Proteins (IGFBPs) }\end{array}$ & $\begin{array}{l}\text { Selective expression in Liver (RPKM } \\
\text { 96.2), Thymus (RPKM 23.3) and } 3 \\
\text { other tissues }\end{array}$ & 7.51 & 0.0004 \\
\hline Pctp & $\begin{array}{l}\text { Phosphatidylcholine transfer } \\
\text { protein }\end{array}$ & $\begin{array}{l}\text { Plays a role in intermembrane transfer of } \\
\text { phosphatidylcholines }\end{array}$ & $\begin{array}{l}\text { Selective expression in Liver (RPKM } \\
\text { 243.0), Testes (RPKM 114.6) and } 8 \\
\text { other tissues }\end{array}$ & 4.00 & 0.0061 \\
\hline MGC116202 & $\begin{array}{l}\text { LOC688736 uncharacterized } \\
\text { protein KIAA0895-like }\end{array}$ & - & $\begin{array}{l}\text { Selective expression in Brain (RPKM } \\
\text { 59.9), Testes (RPKM 34.1) and } 6 \text { other } \\
\text { tissues }\end{array}$ & -7.19 & 0.0073 \\
\hline Ltb4r2 & Leukotriene B4 receptor 2 & $\begin{array}{l}\text { Mouse homolog is a chemoattractant for myeloid } \\
\text { leukocytes }\end{array}$ & $\begin{array}{l}\text { Selective expression in Lung (RPKM } \\
\text { 1.6), Thymus (RPKM 1.1) and } 9 \text { other } \\
\text { tissues }\end{array}$ & 7.11 & 0.0113 \\
\hline Zbtb1 & $\begin{array}{l}\text { Zinc finger and BTB domain } \\
\text { containing } 1\end{array}$ & May bind DNA & $\begin{array}{l}\text { Selective expression in Thymus } \\
\text { (RPKM 102.4), Spleen (RPKM 62.9) } \\
\text { and } 9 \text { other tissues }\end{array}$ & 6.21 & 0.0166 \\
\hline Oxtr & Oxytocin receptor & $\begin{array}{l}\text { G-protein coupled receptor for the peptide hormone, } \\
\text { oxytocin }\end{array}$ & $\begin{array}{l}\text { Selective expression in Adrenal } \\
\text { (RPKM 17.2), Uterus (RPKM 11.7) } \\
\text { and } 7 \text { other tissues }\end{array}$ & -6.81 & 0.0188 \\
\hline Dnajb5 & $\begin{array}{l}\text { DnaJ (Hsp40) homolog, } \\
\text { subfamily B, member } 5\end{array}$ & $\begin{array}{l}\text { Predicted to be involved in chaperone cofactor- } \\
\text { dependent protein refolding and response to unfolded } \\
\text { protein }\end{array}$ & $\begin{array}{l}\text { Selective expression in Muscle (RPKM } \\
\text { 168.1), Brain (RPKM 82.7) and } 9 \text { other } \\
\text { tissues }\end{array}$ & 6.28 & 0.0213 \\
\hline Hspa5 & $\begin{array}{l}\text { Heat shock protein family A } \\
(\text { Hsp70) member } 5\end{array}$ & $\begin{array}{l}\text { secreted protein of the endoplasmic reticulum; may be } \\
\text { involved in the assembly of secreted and membrane- } \\
\text { bound proteins }\end{array}$ & $\begin{array}{l}\text { Selective expression in Liver (RPKM } \\
\text { 2693.8), Heart (RPKM 2015.3) and } 9 \\
\text { other tissues }\end{array}$ & -6.64 & 0.0213 \\
\hline Alox5 & Arachidonate 5-lipoxygenase & $\begin{array}{l}\text { catalyzes the conversion of arachidonate to } \\
\text { leukotriene A4 in leukotriene metabolism }\end{array}$ & $\begin{array}{l}\text { Selective expression in Lung (RPKM } \\
\text { 53.5), Heart (RPKM 25.7) and } 9 \text { other } \\
\text { tissues }\end{array}$ & -6.57 & 0.0213 \\
\hline Dxo & Decapping exoribonuclease & $\begin{array}{l}\text { Hydrolyzes the nicotinamide adenine dinucleotide } \\
\text { (NAD) cap from a subset of RNAs }\end{array}$ & $\begin{array}{l}\text { Selective expression in Adrenal } \\
\text { (RPKM 116.7), Kidney (RPKM 46.2) } \\
\text { and } 9 \text { other tissues }\end{array}$ & 6.59 & 0.0213 \\
\hline
\end{tabular}

Abbreviations: RPKM, reads per kilobase per million mapped reads (mean values given).

during 6 weeks of PC progression (representing stage 1 to stage 3 / 4 PC development)-REV group received Post-RUN EVs from runners, SEV group-EVs from sedentary rats, PBS group-vehicle only (Figure 1).

Six weeks after implantation, tumor masses were found in the ventral prostate of all rats. Noteworthy, no suffering was observed till the endpoint for the majority of tumor-bearing animals. However, two rats from each group were humanely euthanized before the set endpoint of 6 weeks due to signs of suffering. Postmortem analyses revealed that all the animals had tumor masses developed outside the original injection site leading to the stricture of the urethra, a common complication seen in orthotopic rodent PC models [our 
TABLE 2 | Top 10 differentially expressed genes in Pre-RUN vs. sedentary control EVs.

\begin{tabular}{|c|c|c|c|c|c|}
\hline Gene name & Description & Function Smith et al. (2020) & Expression Yu et al. (2014) & LogFC & $\begin{array}{c}\text { Adjusted } \\
p \text {-value }\end{array}$ \\
\hline Dnajb5 & $\begin{array}{l}\text { DnaJ heat shock protein family } \\
\text { (Hsp40) member B5 }\end{array}$ & $\begin{array}{l}\text { Predicted to be involved in chaperone cofactor- } \\
\text { dependent protein refolding and response to } \\
\text { unfolded protein }\end{array}$ & $\begin{array}{l}\text { Selective expression in Muscle (RPKM } \\
\text { 168.1), Brain (RPKM 82.7) and } 9 \text { other } \\
\text { tissues }\end{array}$ & -7.95 & 0.00003 \\
\hline Ripor3 & RIPOR family member 3 & $\begin{array}{l}\text { Orthologous to human RIPOR3, enables protein } \\
\text { binding }\end{array}$ & $\begin{array}{l}\text { Selective expression in Kidney (RPKM } \\
\text { 21.2), Liver (RPKM 17.8) and } 9 \text { other } \\
\text { tissues }\end{array}$ & -7.46 & 0.00003 \\
\hline Poglut1 & Protein O-glucosyltransferase 1 & Involved in the pathway protein glycosylation & $\begin{array}{l}\text { Selective expression in Thymus } \\
\text { (RPKM 128.2), Brain (RPKM 117.2) } \\
\text { and } 9 \text { other tissues }\end{array}$ & 8.21 & 0.00004 \\
\hline Focad & Focadhesin & $\begin{array}{l}\text { Predicted to be located in focal adhesion. } \\
\text { Orthologous to human FOCAD }\end{array}$ & $\begin{array}{l}\text { Selective expression in Brain (RPKM } \\
\text { 101.0), Muscle (RPKM 96.0) and } 9 \\
\text { other tissues }\end{array}$ & -4.91 & 0.0027 \\
\hline Gpx3 & Glutathione peroxidase 3 & $\begin{array}{l}\text { Catalyze the reduction of organic hydroperoxides } \\
\text { and hydrogen peroxide }(\mathrm{H} 2 \mathrm{O} 2) \text { by glutathione, } \\
\text { and thereby protect cells against oxidative } \\
\text { damage }\end{array}$ & $\begin{array}{l}\text { Expression restricted to heart (RPKM } \\
\text { 20.0), kidney (RPKM 587.3), lung } \\
\text { (RPKM 25.6) }\end{array}$ & -6.97 & 0.0032 \\
\hline AC107331.1 & - & $\begin{array}{l}\text { Predicted to enable G protein-coupled receptor } \\
\text { activity and olfactory receptor activity }\end{array}$ & - & -5.16 & 0.0032 \\
\hline RGD1307929 & Similar to CG14967-PA & $\begin{array}{l}\text { Orthologous to human KIAA0100, May be } \\
\text { involved in membrane trafficking }\end{array}$ & $\begin{array}{l}\text { Selective expression in Kidney (RPKM } \\
\text { 513.2), Adrenal (RPKM 381.1) and } 9 \\
\text { other tissues }\end{array}$ & 5.89 & 0.0032 \\
\hline Lpin2 & Lipin 2 & $\begin{array}{l}\text { Predicted to be involved in several processes, } \\
\text { including cellular response to insulin stimulus; fatty } \\
\text { acid catabolic process; and triglyceride } \\
\text { biosynthetic process }\end{array}$ & $\begin{array}{l}\text { Selective expression in Kidney (RPKM } \\
\text { 124.7), Spleen (RPKM 111.6) and } 9 \\
\text { other tissues }\end{array}$ & -5.46 & 0.0032 \\
\hline Tap1 & $\begin{array}{l}\text { Transporter } 1 \text {, ATP binding } \\
\text { cassette subfamily B member }\end{array}$ & $\begin{array}{l}\text { May transport antigenic peptides across the } \\
\text { endoplasmic reticulum membrane in preparation } \\
\text { for MHC class I presentation }\end{array}$ & $\begin{array}{l}\text { Selective expression in Thymus } \\
\text { (RPKM 337.5), Spleen (RPKM 282.5) } \\
\text { and } 9 \text { other tissues }\end{array}$ & -6.65 & 0.0046 \\
\hline Agxt2 & $\begin{array}{l}\text { Alanine-glyoxylate } \\
\text { aminotransferase } 2\end{array}$ & $\begin{array}{l}\text { Predicted to enable alanine-glyoxylate } \\
\text { transaminase activity and beta-alanine-pyruvate } \\
\text { transaminase activity }\end{array}$ & $\begin{array}{l}\text { Expression restricted to liver (RPKM } \\
\text { 29.2) and kidney (RPKM 110.3) }\end{array}$ & -6.28 & 0.0078 \\
\hline
\end{tabular}

Abbreviations: RPKM, reads per kilobase per million mapped reads (mean values given).

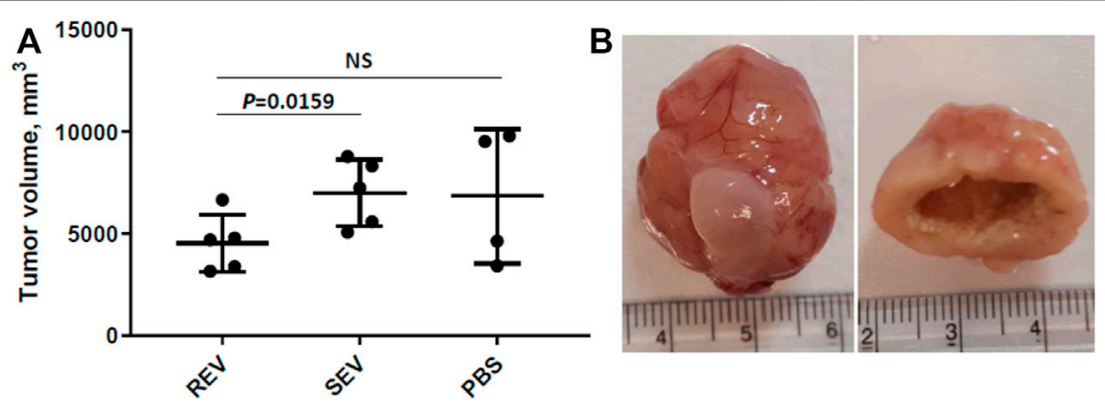

FIGURE 4 | Effects of exercise-induced EVs on the progression of prostate cancer. (A) Comparison of primary tumor volumes between groups after 6 weeks of EV or vehicle administration. In the dot plot, mean tumor volumes are shown, whiskers represent standard deviation; dots represent individual animals. Mann-Whitney test was performed to assess the statistical significance of the differences between groups. (B) A representative example of an orthotopic PLS10 cell-induced prostate tumor (with bladder) and necrosis found in the center of most tumors.

unpublished data (Suzuki et al., 2015)]. These animals were excluded from the data analyses.

Primary tumor volume was assessed as the primary outcome measure and compared between the groups. The mean primary tumor volumes were $4532 \pm 1396 \mathrm{~mm}^{3}$ in the REV group, $7000 \pm$ $1882 \mathrm{~mm}^{3}$ in SEV group, and $6841 \pm 3291 \mathrm{~mm}^{3}$ in PBS group (Figure 4A). Statistically significant differences were seen only when comparing mean tumor volume of rats that received runner-derived EVs and those of SEV group animals (reduction of the primary tumor volume in REV group by $35 \%$, Mann-Whitney $p=0.0159$ ), but the difference was insignificant when compared to the PBS group, due to the small number of animals and large variation in tumor size in this group. All the primary tumors were found to have profound necrosis in the center of the tumor (Figure 4B).

Apart from tumor volumes, the location and number of macroscopic metastatic lesions were assessed. The results were highly inconsistent between individual animals and groups-metastases were found in various locations, including 
TABLE 3 | Overview of the detected macrometastases in F344 PC model rats.

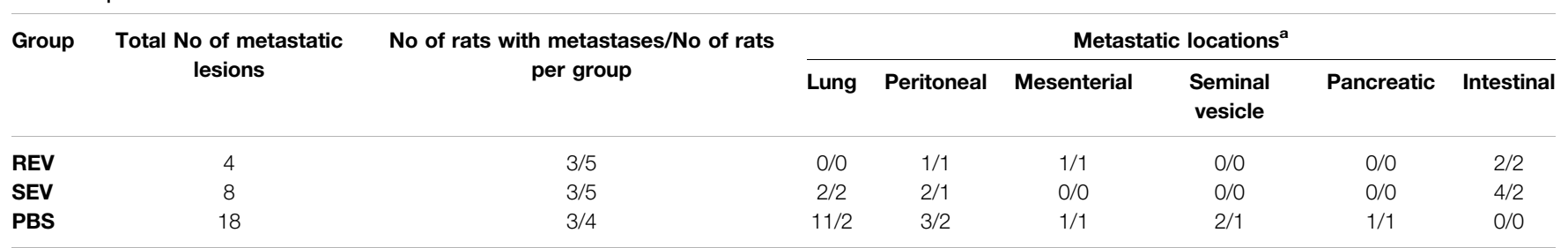

${ }^{a}$ The number of total metastatic lesions in the given location/the number of animals with metastases in the given location.

seminal vesicles, mesenteric, iliac and intestinal lymph nodes, and lungs (Table 3) and revealed no statistically significant differences between any of the study groups in terms of numbers or locations. However, distal lung metastases were observed only in the animals from control groups, but not rats receiving Post-RUN EVs (REV group) implying for a possible protective role of the EVs in cancer progression.

\section{DISCUSSION}

As several previous studies had demonstrated that exercise increases the levels of circulating EVs both in human subjects and laboratory animals (Fruhbeis et al., 2015; Bei et al., 2017; Oliveira et al., 2018; Whitham et al., 2018; Neuberger et al., 2021), we assessed the EV concentration in rat plasma samples collected before and immediately after forced 1-h wheel running exercise. Although the EV levels were increased in some animals, the mean EV concentration and size were not significantly different in the PreRUN and Post-RUN plasma samples. These results are in line with several other studies finding no significant changes in the EV concentration after the exercise (Lovett et al., 2018; Hou et al., 2019; Yoon et al., 2021). This controversy suggests that different types, intensity and duration of exercise may affect the kinetics of EV release from various cell types or their clearance from the circulation in different ways. It could be possible that the induction of EV release during wheel running exercise is too slow to observe a substantial increase after $1 \mathrm{~h}$ or is compensated by increased clearance rate. Such a version is supported by Fruhbeis et al. showing that small EV levels increased immediately after cycling exercise and declined within $90 \mathrm{~min}$ at rest, whereas the increase was moderate but more sustained in response to treadmill running (Fruhbeis et al., 2015). Alternatively, these results may be affected by the use of different EV isolation and quantification methods and the variability of these methods may obscure a moderate increase in EV levels. In this study, we used IZON qEVoriginal $/ 35 \mathrm{~nm}$ columns that have an optimum recovery range of $35-350 \mathrm{~nm}$, thus larger microvesicles may be underrepresented in our EV preps, whereas co-isolation of larger lipoprotein particles is possible. Indeed, TEM images revealed small but consistent co-isolation of lipoprotein particles and the presence of some large particles that may represent lipoprotein aggregates, which may affect the NTA measurements. Hence, immunoisolation of EV subpopulations using EV surface markers may give a better insight into the dynamics of $\mathrm{EV}$ release and the cell types contributing to the pool of circulating EVs (Brahmer et al., 2019; Rigamonti et al., 2020).
Despite the fact that we did not observe a consistent increase of the circulating EV levels during exercise, RNA sequencing analysis revealed substantial differences in the RNA cargo of Pre-RUN and Post-RUN EVs from exercised rats, thus suggesting that EVs were actively released in the circulation in response to the forced wheel running exercise. Although selective RNA sorting mechanisms have been described that bias the RNA profiles of EVs (O'Brien et al., 2020), at least partially the molecular composition of EVs reflect that of the parental cell and therefore the analysis of RNA cargo may reveal the cell types producing EVs during the exercise. All of the 20 DEGs altered in the Post-RUN EVs were protein-coding genes. According to rat transcriptomic BodyMap (Yu et al., 2014), most of the genes that are upregulated in the Post-RUN EVs have tissue-selective or restricted expression pattern with the highest expression levels in the liver, testis, lung, muscle, brain, thymus and kidney. At least in human blood, the major sources of cell-free RNAs are blood cells, followed by much smaller fractions derived from spleen, liver and other tissues (Larson et al., 2021). Our data suggest that fractions of cell-free RNAs derived from organs involved in exercise are increased during the exercise relatively to the blood cell-derived fraction.

The differentially expressed genes in the Post-RUN EVs reflect the physiological processes that are triggered in various cell types in response to exercise. For example, 3 DEGs-Notum (palmitoleoylprotein carboxylesterase), Pctp (phosphatidylcholine transfer protein) and Cyp4b1 (cytochrome P450, family 4, subfamily b, polypeptide 1), which are induced in Post-RUN EVs, are implicated in various metabolic processes. Two other DEGs-Dnajb5 (Dna) heat shock protein family (Hsp40) member B5) and Hspa5 (heat shock protein family A (Hsp70) member 5) are molecular chaperones that are involved in maturation, re-folding and degradation of proteins and play pivotal roles in cell survival under various stress conditions (Archer et al., 2018). Another set of genes is involved in the regulation of immune responses. Ltb4r2 (leukotriene B4 receptor 2) and Alox5 (arachidonate 5lipoxygenase) are involved in leukotriene signaling that play important role in acute and chronic inflammation (Jo-Watanabe et al., 2019), while Zbtb1 (zinc finger and BTB domain containing 1) is a transcription factor that is essential for the development, differentiation and effector function of $\mathrm{T}$ cells (Cheng et al., 2021). Fcrlb (Fc receptor-like $\mathrm{B}$ ) is an $\mathrm{F}_{\mathrm{C}}$ receptor homolog expressed as an intracellular protein in germinal center B cells (Wilson and Colonna, 2005).

We did not find significantly altered miRNAs, when compared the Post-RUN EVs with the Pre-RUN EVs. On the contrary, a recent study by Oliveira et al. found 12 miRNAs that were differentially expressed in rat serum EVs in response to 
treadmill running (Oliveira et al., 2018). The levels of eight of these miRNAs (rno-miR-128-3p, 103-3p, 148a-3p, 191a-5p, 10b5p, 93-5p, 25-3p and 142-5p) were also altered in the Post-RUN EVs in our study, however, the difference did not reach the statistical significance.

Comparison of Pre-RUN EVs from exercised rats with EVs from sedentary control rats revealed 52 DEGs thus showing that exercise has a long-lasting effect on the circulating EV RNA cargo. Among the genes downregulated in the EVs from exercised rats were Gpx3 (glutathione peroxidase 3), $P x d n$ (peroxidasin) and Selenof (selenoprotein F) that are associated with the molecular function "oxidoreductase activity, acting on peroxide as acceptor"; two of them-Gpx3 and Selenof are also associated with "selenium binding". Although several studies have shown that the levels of oxidative stress markers such as glutathione peroxidase increase during the exercise (Khcharem et al., 2021; Mesquita et al., 2021), our results suggest that their baseline levels are lower in exercised than in sedentary animals. Among the DEGs were two miRNAs-miR378b and miR35, that were present in the runner EVs but undetectable in the sedentary rat EVs. Both of them are implicated in the regulation of various processes in the body, including insulin sensitivity (Li et al., 2020) and protection against insulin resistance (Chen et al., 2019).

Several previous studies have investigated the effects of exercise on tumor incidence and progression in animal models. Nilsson et al. studied the effects of early-onset, lifelong voluntary wheel running in a naturally aging mouse model and showed that exercise protected against multiple types of cancer, lowered systemic inflammation and extended the health-span of naturally aged mice (Nilsson et al., 2019). Another study showed that voluntary wheel running reduced tumor incidence and growth across 5 different tumor models, including subcutaneous injection of B16F10 murine melanoma cells, intravenous injection of B16F10 melanoma cells, diethylnitrosamine (DEN) - induced liver cancer, Lewis Lung carcinoma model, and $\mathrm{Tg}(\mathrm{Grm} 1) \mathrm{EPv}$ transgenic male mice model. In the subcutaneous B16 models, tumors from running mice showed higher infiltration of NK cells, $\mathrm{CD}^{+} \mathrm{T}$ cells and dendritic cells, while the metastasis model had infiltrated NK cells only (Pedersen et al., 2016), suggesting that the mobilization or increased cytotoxicity of NK cells may be one of the mechanisms of action.

Here, we for the first time studied the effects of exercise-induced EVs on the progression of cancer in a rat model of metastatic prostate cancer. Results showed that regular injections of exerciseinduced EVs into tumor-bearing rats reduced the primary tumor growth by $\sim 35 \%$ and possibly may have delayed the development of lung metastasis. Pedersen et al. proposed four key mechanisms implicated in exercise-mediated cancer protection: normalization of tumor vasculature and blood flow, boosting immune cell functions, reprogramming metabolic pathways in cancer cells and controlling tumor growth by bioactive molecules secreted by skeletal muscle during contraction (Pedersen et al., 2015). In principle, exercise-induced EVs may contribute to all of these mechanisms, however, what is the scale of their contribution and which of the mechanisms dominate in the exercise-induced EV-mediated cancer protection remains to be established.

A shortcoming of our study, however, is the small sample size that remained after excluding animals showing the signs of suffering and large variation of the obtained data, therefore the results of this exploratory study should be regarded as indicative and should be validated in a larger cohort of animals. Moreover, the biodistribution, uptake and intracellular fate of the exercise-induced EVs and the effects triggered in the recipient cells remains to be investigated.

In summary, we show that the RNA cargo of EVs released into the circulation during exercise is altered as compared to the resting state and provide evidence that exercise-induced EVs may modulate tumor physiology and delay the progression of cancer thus supporting the idea that regular physical exercise should be prescribed to prostate cancer patients as a tertiary prevention measure.

\section{DATA AVAILABILITY STATEMENT}

The datasets presented in this study can be found in online repositories. The names of the repository/repositories and accession number(s) can be found below: https://www.ebi.ac. uk/arrayexpress/, E-MTAB-11020.

\section{ETHICS STATEMENT}

The animal study was reviewed and approved by National animal welfare and ethics committee (permit no. 121/2021).

\section{AUTHOR CONTRIBUTIONS}

AL, ZK and LiS designed research, LiS, JA, LK, NR, and ZK performed research and participated in the analysis and interpretation of the results, PZ performed the RNAseq data analysis and statistical analyses. AL, LiS, JA, LK, and ZK wrote the manuscript. MK and LaS helped with animal surgery and postsurgical care. ST provided the cell line. All authors have read and approved the manuscript.

\section{FUNDING}

This work was funded by the Latvian Council of Science, Project No. lzp-2018/0269.

\section{ACKNOWLEDGMENTS}

We are grateful to Prof. Baiba Jansone form Department of Pharmacology, University of Latvia for providing us with the forced running wheel system for rats.

\section{SUPPLEMENTARY MATERIAL}

The Supplementary Material for this article can be found online at: https://www.frontiersin.org/articles/10.3389/fmolb.2021.784080/ full\#supplementary-material 


\section{REFERENCES}

Archer, A. E., Von Schulze, A. T., and Geiger, P. C. (2018). Exercise, Heat Shock Proteins and Insulin Resistance. Philos. Trans. R Soc. Lond. B Biol. Sci. 373 (1738). 20160529. doi:10.1098/rstb.2016.0529

Axtell, M. J. (2013). ShortStack: Comprehensive Annotation and Quantification of Small RNA Genes. RNA 19, 740-751. doi:10.1261/rna.035279.112

Bei, Y., Xu, T., Lv, D., Yu, P., Xu, J., Che, L., et al. (2017). Exercise-induced Circulating Extracellular Vesicles Protect against Cardiac IschemiaReperfusion Injury. Basic Res. Cardiol. 112, 38. doi:10.1007/s00395-0170628-Z

Belloni, S., Arrigoni, C., and Caruso, R. (2021). Effects from Physical Exercise on Reduced Cancer-Related Fatigue: a Systematic Review of Systematic Reviews and Meta-Analysis. Acta Oncol. 1, 1-10. doi:10.1080/0284186x.2021.1962543

Bourke, L., Smith, D., Steed, L., Hooper, R., Carter, A., Catto, J., et al. (2016). Exercise for Men with Prostate Cancer: A Systematic Review and MetaAnalysis. Eur. Urol. 69, 693-703. doi:10.1016/j.eururo.2015.10.047

Brahmer, A., Neuberger, E., Esch-Heisser, L., Haller, N., Jorgensen, M. M., Baek, R., et al. (2019). Platelets, Endothelial Cells and Leukocytes Contribute to the Exercise-Triggered Release of Extracellular Vesicles into the Circulation. J. Extracell. Vesicles 8, 1615820. doi:10.1080/ 20013078.2019.1615820

Buchner, A., Erdfelder, E., Faul, F., and Lang, A.-G. (2021). G*Power. Statistical Power Analyses For Mac And Windows [Online]. Heinrich-Heine-Universität Düsseldorf. Available at: https://www.psychologie.hhu.de/arbeitsgruppen/ allgemeine-psychologie-und-arbeitspsychologie/gpower (Accessed 09 8, 2021).

Carlson, M. (2019a). GO.db: A Set of Annotation Maps Describing the Entire Gene Ontology. R package version 3.8.2. doi:10.18129/B9.bioc.GO.db

Carlson, M. (2019b). org.Rn.eg.db: Genome Wide Annotation for Rat. R package version 3.8.2. doi:10.18129/B9.bioc.org.Rn.eg.db

Caruso, S., and Poon, I. K. H. (2018). Apoptotic Cell-Derived Extracellular Vesicles: More Than Just Debris. Front. Immunol. 9, 1486. doi:10.3389/ fimmu.2018.01486

Chen, S. H., Liu, X. N., and Peng, Y. (2019). MicroRNA-351 Eases Insulin Resistance and Liver Gluconeogenesis via the PI3K/AKT Pathway by Inhibiting FLOT2 in Mice of Gestational Diabetes Mellitus. J. Cel Mol. Med. 23, 5895-5906. doi:10.1111/jcmm.14079

Cheng, Z.-Y., He, T.-T., Gao, X.-M., Zhao, Y., and Wang, J. (2021). ZBTB Transcription Factors: Key Regulators of the Development, Differentiation and Effector Function of T Cells. Front. Immunol. 12, 713294. doi:10.3389/ fimmu.2021.713294

Endzeliṇš, E., Berger, A., Melne, V., Bajo-Santos, C., Soboḷevska, K., Ābols, A., et al. (2017). Detection of Circulating miRNAs: Comparative Analysis of Extracellular Vesicle-Incorporated miRNAs and Cell-free miRNAs in Whole Plasma of Prostate Cancer Patients. BMC Cancer 17, 730. doi:10.1186/s12885017-3737-z

Falcon, S., and Gentleman, R. (2007). Using GOstats to Test Gene Lists for GO Term Association. Bioinformatics 23, 257-258. doi:10.1093/bioinformatics/ bt1567

Ferlay, J., Soerjomataram, I., Dikshit, R., Eser, S., Mathers, C., Rebelo, M., et al. (2015). Cancer Incidence and Mortality Worldwide: Sources, Methods and Major Patterns in GLOBOCAN 2012. Int. J. Cancer. 136, E359-E386. doi:10.1002/ijc.29210

Frühbeis, C., Helmig, S., Tug, S., Simon, P., and Krämer-Albers, E.-M. (2015). Physical Exercise Induces Rapid Release of Small Extracellular Vesicles into the Circulation. J. Extracell. Vesicles 4, 28239. doi:10.3402/jev.v4.28239

Hojman, P., Dethlefsen, C., Brandt, C., Hansen, J., Pedersen, L., and Pedersen, B. K. (2011). Exercise-induced Muscle-Derived Cytokines Inhibit Mammary Cancer Cell Growth. Am. J. Physiol. Endocrinol. Metab. 301, E504-E510. doi:10.1152/ ajpendo.00520.2010

Hou, Z., Qin, X., Hu, Y., Zhang, X., Li, G., Wu, J., et al. (2019). Longterm ExerciseDerived Exosomal miR-342-5p. Circ. Res. 124, 1386-1400. doi:10.1161/ circresaha.118.314635

Idorn, M., and Thor Straten, P. (2017). Exercise and Cancer: from "healthy" to "therapeutic". Cancer Immunol. Immunother. 66, 667-671. doi:10.1007/s00262017-1985-z
Jo-Watanabe, A., Okuno, T., and Yokomizo, T. (2019). The Role of Leukotrienes as Potential Therapeutic Targets in Allergic Disorders. Int. J. Mol. Sci. 20, 3580. doi:10.3390/ijms20143580

Johnstone, R. M., Adam, M., Hammond, J. R., Orr, L., and Turbide, C. (1987). Vesicle Formation during Reticulocyte Maturation. Association of Plasma Membrane Activities with Released Vesicles (Exosomes). J. Biol. Chem. 262, 9412-9420. doi:10.1016/s0021-9258(18)48095-7

Kenfield, S. A., Stampfer, M. J., Giovannucci, E., and Chan, J. M. (2011). Physical Activity and Survival after Prostate Cancer Diagnosis in the Health Professionals Follow-Up Study. J. Clin. Oncol. 29, 726-732. doi:10.1200/ jco.2010.31.5226

Khcharem, A., Souissi, M., Atheymen, R., Souissi, W., and Sahnoun, Z. (2021). Acute Caffeine Ingestion Improves 3-km Run Performance, Cognitive Function, and Psychological State of Young Recreational Runners. Pharmacol. Biochem. Behav. 207, 173219. doi:10.1016/ j.pbb.2021.173219

Kozomara, A., Birgaoanu, M., and Griffiths-Jones, S. (2019). miRBase: from microRNA Sequences to Function. Nucleic Acids Res. 47, D155-D162. doi:10.1093/nar/gky1141

Langmead, B., and Salzberg, S. L. (2012). Fast Gapped-Read Alignment with Bowtie 2. Nat. Methods 9, 357-359. doi:10.1038/nmeth.1923

Larson, M. H., Pan, W., Kim, H. J., Mauntz, R. E., Stuart, S. M., Pimentel, M., et al. (2021). A Comprehensive Characterization of the Cell-free Transcriptome Reveals Tissue- and Subtype-specific Biomarkers for Cancer Detection. Nat. Commun. 12, 2357. doi:10.1038/s41467-021-22444-1

Li, Y.-y., Zhong, Y.-j., Cheng, Q., Wang, Y.-z., Fan, Y.-y., Yang, C.-f., et al. (2020). miR-378b Regulates Insulin Sensitivity by Targeting Insulin Receptor and P110a in Alcohol-Induced Hepatic Steatosis. Front. Pharmacol. 11, 717. doi:10.3389/fphar.2020.00717

Liao, Y., Smyth, G. K., and Shi, W. (2019). The R Package Rsubread Is Easier, Faster, Cheaper and Better for Alignment and Quantification of RNA Sequencing Reads. Nucleic Acids Res. 47, e47. doi:10.1093/nar/gkz114

Lovett, J. A. C., Durcan, P. J., and Myburgh, K. H. (2018). Investigation of Circulating Extracellular Vesicle MicroRNA Following Two Consecutive Bouts of Muscle-Damaging Exercise. Front. Physiol. 9, 1149. doi:10.3389/ fphys.2018.01149

Mähler, M., Berard, M., Feinstein, R., Gallagher, A., Illgen-Wilcke, B., PritchettCorning, K., et al. (2014). FELASA Recommendations for the Health Monitoring of Mouse, Rat, Hamster, guinea Pig and Rabbit Colonies in Breeding and Experimental Units. Lab. Anim. 48, 178-192. doi:10.1177/ 0023677213516312

Martin, M. (2011). Cutadapt Removes Adapter Sequences from High-Throughput Sequencing Reads. EMBnet J. 17, 10-12. doi:10.14806/ej.17.1.200

Mccarthy, D. J., Chen, Y., and Smyth, G. K. (2012). Differential Expression Analysis of Multifactor RNA-Seq Experiments with Respect to Biological Variation. Nucleic Acids Res. 40, 4288-4297. doi:10.1093/nar/gks042

Mesquita, P. H. C., Lamb, D. A., Godwin, J. S., Osburn, S. C., Ruple, B. A., Moore, J. H., et al. (2021). Effects of Resistance Training on the Redox Status of Skeletal Muscle in Older Adults. Antioxidants (Basel) 10 (3), 350. doi:10.3390/ antiox 10030350

Moore, S. C., Lee, I.-M., Weiderpass, E., Campbell, P. T., Sampson, J. N., Kitahara, C. M., et al. (2016). Association of Leisure-Time Physical Activity with Risk of 26 Types of Cancer in 1.44 Million Adults. JAMA Intern. Med. 176, 816-825. doi:10.1001/jamainternmed.2016.1548

Muller, L., Mitsuhashi, M., Simms, P., Gooding, W. E., and Whiteside, T. L. (2016). Tumor-derived Exosomes Regulate Expression of Immune Function-Related Genes in Human T Cell Subsets. Sci. Rep. 6, 20254. doi:10.1038/srep20254

Muller, L., Simms, P., Hong, C.-S., Nishimura, M. I., Jackson, E. K., Watkins, S. C., et al. (2017). Human Tumor-Derived Exosomes (TEX) Regulate Treg Functions via Cell Surface Signaling rather Than Uptake Mechanisms. Oncoimmunology 6, e1261243. doi:10.1080/2162402x.2016.1261243

Nakanishi, H., Takeuchi, S., Kato, K., Shimizu, S., Kobayashi, K., Tatematsu, M., et al. (1996). Establishment and Characterization of Three Androgenindependent, Metastatic Carcinoma Cell Lines from 3,2'-Dimethyl-4Aminobiphenyl-Induced Prostatic Tumors in F344 Rats. Jpn. J. Cancer Res. 87, 1218-1226. doi:10.1111/j.1349-7006.1996.tb03136.x 
Neuberger, E. W. I., Hillen, B., Mayr, K., Simon, P., Krämer-Albers, E. M., and Brahmer, A. (2021). Kinetics and Topology of DNA Associated with Circulating Extracellular Vesicles Released during Exercise. Genes (Basel) 12, 522. doi:10.3390/genes12040522

Nilsson, M. I., Bourgeois, J. M., Nederveen, J. P., Leite, M. R., Hettinga, B. P., Bujak, A. L., et al. (2019). Lifelong Aerobic Exercise Protects against Inflammaging and Cancer. PLoS One 14, e0210863. doi:10.1371/ journal.pone. 0210863

O'brien, K., Breyne, K., Ughetto, S., Laurent, L. C., and Breakefield, X. O. (2020). RNA Delivery by Extracellular Vesicles in Mammalian Cells and its Applications. Nat. Rev. Mol. Cel Biol. 21, 585-606. doi:10.1038/s41580-0200251-y

Oliveira, G. P., Jr., Porto, W. F., Palu, C. C., Pereira, L. M., Petriz, B., Almeida, J. A., et al. (2018). Effects of Acute Aerobic Exercise on Rats Serum Extracellular Vesicles Diameter, Concentration and Small RNAs Content. Front. Physiol. 9, 532. doi:10.3389/fphys.2018.00532

Pedersen, L., Christensen, J. F., and Hojman, P. (2015). Effects of Exercise on Tumor Physiology and Metabolism. Cancer J. 21, 111-116. doi:10.1097/ ppo.0000000000000096

Pedersen, L., Idorn, M., Olofsson, G. H., Lauenborg, B., Nookaew, I., Hansen, R. H., et al. (2016). Voluntary Running Suppresses Tumor Growth through Epinephrine- and IL-6-Dependent NK Cell Mobilization and Redistribution. Cel Metab. 23, 554-562. doi:10.1016/j.cmet.2016.01.011

Rigamonti, A. E., Bollati, V., Pergoli, L., Iodice, S., De Col, A., Tamini, S., et al. (2020). Effects of an Acute Bout of Exercise on Circulating Extracellular Vesicles: Tissue-, Sex-, and BMI-Related Differences. Int. J. Obes. 44, 1108-1118. doi:10.1038/s41366-019-0460-7

Ruiz-Casado, A., Martín-Ruiz, A., Pérez, L. M., Provencio, M., Fiuza-Luces, C., and Lucia, A. (2017). Exercise and the Hallmarks of Cancer. Trends Cancer 3, 423-441. doi:10.1016/j.trecan.2017.04.007

Shephard, R. J. (2017). Physical Activity and Prostate Cancer: An Updated Review. Sports Med. 47, 1055-1073. doi:10.1007/s40279-016-0648-0

Smith, J. R., Hayman, G. T., Wang, S. J., Laulederkind, S. J. F., Hoffman, M. J., Kaldunski, M. L., et al. (2020). The Year of the Rat: The Rat Genome Database at 20: a Multi-Species Knowledgebase and Analysis Platform. Nucleic Acids Res. 48, D731-D742. doi:10.1093/nar/gkz1041

Suzuki, S., Naiki-Ito, A., Kuno, T., Punfa, W., Long, N., Kato, H., et al. (2015). Establishment of a Syngeneic Orthotopic Model of Prostate Cancer in Immunocompetent Rats. J. Toxicol. Pathol. 28, 21-26. doi:10.1293/tox.20140050

Théry, C., Witwer, K. W., Aikawa, E., Alcaraz, M. J., Anderson, J. D., Andriantsitohaina, R., et al. (2018). Minimal Information for Studies of Extracellular Vesicles 2018 (MISEV2018): a Position Statement of the International Society for Extracellular Vesicles and Update of the
MISEV2014 Guidelines. J. Extracell. Vesicles 7, 1535750. doi:10.1080/ 20013078.2018.1535750

Tkach, M., and Théry, C. (2016). Communication by Extracellular Vesicles: Where We Are and where We Need to Go. Cell 164, 1226-1232. doi:10.1016/ j.cell.2016.01.043

Whitham, M., Parker, B. L., Friedrichsen, M., Hingst, J. R., Hjorth, M., Hughes, W. E., et al. (2018). Extracellular Vesicles Provide a Means for Tissue Crosstalk during Exercise. Cel Metab. 27, 237-251. doi:10.1016/j.cmet.2017.12.001

Wilson, T. J., and Colonna, M. (2005). A New Fc Receptor Homolog, FREB2, Found in Germinal center B Cells. Genes Immun. 6, 341-346. doi:10.1038/ sj.gene.6364185

Winter, D. J. (2017). Rentrez: an R Package for the NCBI eUtils API. $R$ J. 9, 520-526. doi:10.32614/rj-2017-058

Yáñez-Mó, M., Siljander, P. R.-M., Andreu, Z., Bedina Zavec, A., Borràs, F. E., Buzas, E. I., et al. (2015). Biological Properties of Extracellular Vesicles and Their Physiological Functions. J. Extracellular Vesicles 4, 27066. doi:10.3402/jev.v4.27066

Yoon, K. J., Park, S., Kwak, S. H., and Moon, H. Y. (2021). Effects of Voluntary Running Wheel Exercise-Induced Extracellular Vesicles on Anxiety. Front. Mol. Neurosci. 14, 665800. doi:10.3389/fnmol.2021.665800

Yu, Y., Fuscoe, J. C., Zhao, C., Guo, C., Jia, M., Qing, T., et al. (2014). A Rat RNASeq Transcriptomic BodyMap across 11 Organs and 4 Developmental Stages. Nat. Commun. 5, 3230. doi:10.1038/ncomms4230

Zhan, D., Cross, A., Wright, H. L., Moots, R. J., Edwards, S. W., and Honsawek, S. (2021). Internalization of Neutrophil-Derived Microvesicles Modulates TNFaStimulated Proinflammatory Cytokine Production in Human Fibroblast-like Synoviocytes. Int. J. Mol. Sci. 22 (14), 7049. doi:10.3390/ijms22147409

Conflict of Interest: The authors declare that the research was conducted in the absence of any commercial or financial relationships that could be construed as a potential conflict of interest.

Publisher's Note: All claims expressed in this article are solely those of the authors and do not necessarily represent those of their affiliated organizations, or those of the publisher, the editors and the reviewers. Any product that may be evaluated in this article, or claim that may be made by its manufacturer, is not guaranteed or endorsed by the publisher.

Copyright (C) 2022 Sadovska, Auders, Keiša, Romanchikova, Silamik̦ele, Kreišmane, Zayakin, Takahashi, Kalnina and Linē. This is an open-access article distributed under the terms of the Creative Commons Attribution License (CC BY). The use, distribution or reproduction in other forums is permitted, provided the original author(s) and the copyright owner(s) are credited and that the original publication in this journal is cited, in accordance with accepted academic practice. No use, distribution or reproduction is permitted which does not comply with these terms. 